\title{
Spinning a mental web
}

\section{Danielle S. Bassett* and Felix Siebenhühner}

Department of Physics, University of California Santa Barbara, Santa Barbara, CA, USA

*Correspondence: dbassett@physics.ucsb.edu

\section{A book review on}

Networks of the Brain

by Olaf Sporns

In our ever more connected world, the critical importance of networks is becoming increasingly apparent in everything from ecological to financial systems, from social media to electricity grids. It is becoming clear, as recently demonstrated by the financial crisis, that the failure of single components can have drastic consequences for the whole system. (As Sporns drily puts it: "When networks fail, bad things tend to happen"). In order to begin to understand these ripple effects, the features and characteristics of underlying network architectures must be examined.

As Sporns (2010) elegantly propounds in his recent book, the human brain appears to be no exception. He suggests that the brain can be aptly regarded as a network, and that the framework and tools of network science have begun and will continue to provide necessary and timely insights into brain function and mental phenomena. Network science, he argues, is particularly suited to the study of the brain, a complex system composed of many interacting parts which together form structures and facilitate activity patterns that span a variety of spatial and temporal scales.

Key features of brain networks that are highlighted throughout the book include a delicate balance between segregation and integration, instantiated by a modular organization over several hierarchical levels. Modules and submodules can be specialized to perform specific tasks while remaining capable of the adjustment needed to perform new roles as the environment changes, providing the system with both adaptability and robustness. Sporns (2010) emphasizes the central role that anatomical connectivity plays in constraining both resting and taskrelated function, and specifically the role of structural hubs - large-scale brain regions with a preponderance of connections that tend to link disparate modules over large distances - that play a critical part in shaping the brain's routine operations.

While the book covers an astonishing breadth of ideas, it does so by methodically forming a coherent narrative, building on intuitive understanding and drawing from a wide body of research. From the basics of network architectures and small-scale neuroanatomy, we are ushered on to properties of large-scale anatomical structure, its small-world network organization and constraints on its development and evolution. This examination of anatomical scaffolding forms the backdrop for the later chapters on the role of networks in spontaneous brain function, cognition, disease, and development. The book concludes by delving into the theoretical and philosophical: an account of computational models of brain dynamics and the determination of neural complexity is followed by a discussion of the brain's dependence upon and interaction with the immediate body and the further removed environment. On the backdrop of comprehensive research, Sporns (2010) promotes a surprisingly holistic account of brain organization and function, convincingly spinning the picture of a mental web before our eyes.

Sporns (2010) places the relatively young concepts of network science into the broader context of multiple disciplines (e.g., biology, psychology, philosophy, computer science) and multiple time scales (both recent and historical). The historical quotations that headline each chapter clearly demonstrate that many of these concepts are significantly older than we might expect, some reaching back to the nineteenth century or earlier. We begin to see how these concepts can form a framework in which to draw together mankind's knowledge of the brain in particular and understanding of the mind and consciousness more generally. Sporns (2010) emphasizes the central position that the focus on network structure facilitates a broad coalescence of the science of the brain.

"Networks of the Brain" provides a multifaceted intellectual tour in highly readable prose. In some $300+$ pages, Sporns (2010) manages to delve into everything from minute neuroanatomy to large-scale callosal structure, from cancer genes (p53) to amyloid deposits in Alzheimer's disease, from the nematode to Homo sapiens, from computational models and pure theory to detailed experiments. He recounts in-depth histories of concepts from both the distant and more recent past: from graph theory (Euler, 1736) or Turing's (1952) theory of morphogenesis to the more recently proposed relationship between functional and structural connectivity (Koch, 2002). While providing the reader with a broad factual basis, Sporns (2010) also tackles potentially controversial topics such as whether or not the brain has evolved for optimality and the relative importance of theories of neuroreductionism and emergence. The tome is heavily peppered with figures that range from original historical plates to contemporary redrawings of previous work to Sporns' (2010) own detailed constructions, some of them beautifully reprinted in color at the book's center.

This very instructive scientific milieu does not, however, include mathematical descriptions, e.g., of networks, network properties, and the computational models discussed in the later chapters. In doing so, Sporns (2010) aims to make his book understandable to all interested readers. However, those mathematically inclined may utilize the extensive reference list to find original research articles that address 
the mathematics in more detail. For the reader interested in a single reference on the mathematics of networks and network properties, we suggest Newman's (2010) comprehensive book "Networks: An Introduction."

On the whole, Sporns (2010) has done an excellent job making this book understandable and accessible for all intended readers, with or without a great deal of knowledge regarding either networks or neuroscience. Importantly, this achievement is not accomplished by sacrificing either depth or insight. This volume fills an important void by introducing the concept of networks to neuroscientists and neuroscience to network scientists. As such, this book will likely be an important catalyst for new interdisciplinary efforts in this exciting and evolving field.

\section{ACKNOWLEDGMENTS}

This work was supported by the Institute for Collaborative Biotechnologies through contract no. W911NF-09-D-0001 from the U.S. Army Research Office and the Daryl and Marguerite Errett Discovery Award in Biomedical Research (2011).

\section{REFERENCES}

Euler, L. (1736). Solutio problematis ad geometriam situs pertinentis. Comment. Acad. Sci. U. Petrop. 8, 128-140.

Koch, M. A., Norris, D. G., and Hund-Georgiadis, M. (2002). An investigation of functional and anatomi- cal connectivity using magnetic resonance imaging. Neuroimage 16, 241-250.

Newman, M. (2010). Networks: An Introduction. Oxford: Oxford University Press.

Sporns, O. (2010). Networks of the Brain. Cambridge: The MIT Press.

Turing, A. M. (1952). The chemical basis of morphogenesis. Philos. Trans. Roy. Soc. Lond. B. 237, 37-72.

Received: 01 November 2011; accepted: 24 January 2012; published online: 20 February 2012.

Citation: Bassett DS and Siebenhühner F (2012) Spinning a mental web. Front. Hum. Neurosci. 6:13. doi: 10.3389/ fnhum.2012.00013

Copyright (c) 2012 Bassett and Siebenhühner. This is an open-access article distributed under the terms of the Creative Commons Attribution Non Commercial License, which permits non-commercial use, distribution, and reproduction in other forums, provided the original authors and source are credited. 